\title{
Operator Product Expansions and Composite Field Operators in the General Framework of Quantum Field Theory
}

\author{
KENNETH G. WILSON \\ Laboratory of Nuclear Studies, Cornell University, Ithaca, New York \\ WOLFHART ZIMMERMANN* \\ Department of Physics, New York University, New York, N.Y.
}

Received July 26, 1971

\begin{abstract}
The short distance behavior of field operator products is analyzed. It is shown that under certain conditions operator product expansions can be derived which give complete information on the short distance behavior and lead to the construction of composite field operators.
\end{abstract}

\section{Introduction}

A central problem in local quantum field theory is the definition of products of field operators at the same point. Important examples of such composite field operators are Lagrangian densities, energy-momentum tensors, interaction terms of local field equations and current operators associated with internal symmetries. Let $A_{1}, \ldots, A_{n}$ be field operators satisfying the usual postulates of local quantum field theory ${ }^{1}$. The difficulty in constructing composite operators such as

$$
A_{1}(x) \ldots A_{a}(x)
$$

originates in the singularity of the operator product

$$
A_{1}\left(x_{1}\right) \ldots A_{a}\left(x_{a}\right)
$$

for coinciding arguments. Such singularities inevitably occur as a consequence of relativistic invariance and positive definite metric in Hilbert space [1].

In Ref. [2] an expansion of the operator product (1.2) was proposed which exhibits the singularities near $x_{j}=x$ and simultaneously allows

* Supported in parts by funds from the National Science Foundation Grant No. GP-25609.

${ }^{1}$ Each of the operators $A_{j}$ may have several components transforming like a tensor under homogeneous Lorentz transformations.

7 Commun. math. Phys., Vol. 24 
the construction of composite operators. The hypothesis is that any operator product (1.2) may be expanded in the form ${ }^{2}$

$$
A_{1}\left(x+\xi_{1}\right) \ldots A_{a}\left(x+\xi_{a}\right)=\sum_{k=1}^{n} f_{k}\left(\xi_{1}, \ldots, \xi_{a}\right) B_{k}(x)+R\left(x, \xi_{1}, \ldots, \xi_{a}\right)
$$

where the remainder $R$ vanishes at $\xi_{j}=0$ while the functions $f_{k}$ become singular (or non-vanishing) in the limit $\xi_{j} \rightarrow 0$. The operators $B_{k}$ are local and may be regarded as composite field operators associated with the formal product (1.1).

In Ref. [3] this hypothesis was generalized by assuming that any operator product (1.2) may be represented as a series

$$
A_{1}\left(x+\xi_{1}\right) \ldots A_{a}\left(x+\xi_{a}\right)=\sum_{k=1}^{\infty} f_{k}\left(\xi_{1}, \ldots, \xi_{a}\right) B_{k}(x) .
$$

The series is asymptotic in the sense that the coefficients

$$
f_{k}\left(\varrho \lambda_{1}, \ldots, \varrho \lambda_{a}\right)
$$

vanish stronger than $\varrho^{N}$ for $k \geqq k(N)$ provided $k(N)$ is chosen large enough. In addition dimensional rules were given in Ref. $[2,3]$ which provide detailed information on the operators $B_{k}$ and the singularities of the coefficients $f_{k}$.

We summarize the results concerning operator product expansions and the properties of composite field operators which so far have been obtained in the perturbation theory of renormalizable interactions, and exactly for lower dimensional models. Local field equations with properly defined interaction terms were first introduced by Valatin [4] and have been verified in all orders of perturbation theory $[5,6]$. Independent of perturbation theory Glimm and Jaffe have derived local field equations for the model of $\phi^{4}$-coupling in two dimensions [7]. Similar results have been obtained for other twodimensional models $[8,9]^{3}$. The principal parts (1.3) of operator products were derived by Brandt [5], moreover the asymptotic expansion (1.4) has been confirmed in perturbation theory $[10,11]$. Operator product expansions and composite operators have been studied in detail for the Thirring model [8]. In perturbation theory a definition of composite operators can easily be given by applying Bogoliubov's renormalization technique to the Gell-Mann Low expansion of the relevant Green's functions. Under certain conditions it has been possible to define current operators which have the required properties, such as conservation laws, commutation relations, etc. $[10,12]$. In

\footnotetext{
2 The product $f_{k} B_{k}$ may include a sum over some Lorentz indices of $f_{k}$ and $B_{k}$.

${ }^{3}$ Earlier references can be found in Ref. [11].
} 
particular it has been shown that in a renormalizable theory it is always possible to construct an energy-momentum tensor $[10,12]$.

The purpose of this paper is to show how an operator product expansion can be derived from general principles. We begin with a brief outline of the method, leaving questions of mathematical rigor for the detailed discussion of the following sections. Our aim is to

(i) define local operators associated with the formal product (1.1),

(ii) to completely analyze the asymptotic behaviour of (1.2) for $x_{j} \rightarrow x$. We introduce fixed vectors and a scaling parameter $\varrho$ by

$$
x_{j}=x+\varrho \lambda_{j}(\eta), \quad \lambda_{j}(\eta)=\sum_{i=1}^{a-1} C_{j i} \eta_{i}
$$

with

$$
\frac{\partial\left(x_{1} \ldots x_{a}\right)}{\partial\left(x \eta_{1} \ldots \eta_{a-1}\right)} \neq 0 \text { for } \varrho \neq 0
$$

and consider the operator product (1.2) as a function of $x, \eta_{j}$ and $\varrho$

$$
\begin{aligned}
P(x, \eta, \varrho) & =A\left(x_{1}\right) \ldots A\left(x_{a}\right) \\
\eta & =\left(\eta_{1}, \ldots, \eta_{a-1}\right) .
\end{aligned}
$$

If $P$ diverges for $\varrho \rightarrow 0$ we define an operator

$$
C_{1}(x \eta)=\lim _{\varrho \rightarrow 0} \frac{P(x \eta \varrho)}{f_{1}(\varrho)}
$$

dividing $P$ by a suitable function $f_{1}$. The singularity of $f_{1}$ is restricted by the condition that the result be finite and different from zero. A suitable $f_{1}$ can be found if we make the assumption that there are "most singular" matrix elements

$$
(\tilde{\Phi}, P(\tilde{x} \tilde{\eta} \varrho) \tilde{\Psi})
$$

of $P$. A matrix element (1.8) is called most singular if it is as singular or more singular than any other matrix element of $P$ near $\varrho=0$. Choosing one of the matrix elements (1.8) as the function $f_{1}$ the operator (1.7) will be finite and not identically zero. Evidently $C_{1}$ is a local operator. In many cases $C_{1}$ turns out to be a multiple of the identity [13]. However, more composite operators can be found by further examining the behavior of $P(x \eta \varrho)$ for $\varrho \rightarrow 0$. Defining an operator $P_{2}$ by

$$
P(x \eta \varrho)=f_{1}(\varrho) C_{1}(x \eta)+P_{2}(x \eta \varrho)
$$

we have

$$
\lim _{\varrho \rightarrow 0} \frac{P_{2}}{f_{1}}=0
$$


as follows from (1.7). If

$$
\lim _{\varrho \rightarrow 0} P_{2}=0
$$

(1.9) already gives complete information on the singularities of $P$ near $\varrho=0$. If, however, some of the matrix elements of $P_{2}$ diverge at $\varrho=0$ the product $P$ has additional singularities. In this case we repeat the procedure for $P_{2}$ and obtain

where

$$
P_{2}(x \eta \varrho)=f_{2}(\varrho) C_{2}(x \eta)+P_{3}(x \eta \varrho)
$$

$$
C_{2}(x \eta)=\lim _{\varrho \rightarrow 0} \frac{P_{2}(x \eta \varrho)}{f_{2}(\varrho)}
$$

is a local, non-vanishing field operator. The function $f_{2}$ is one of the most singular matrix elements of $P_{2}$. It diverges in the limit

$$
\lim _{\varrho \rightarrow 0} f_{2}(\varrho)=\infty,
$$

but is less singular than $f_{1}$

$$
\lim _{\varrho \rightarrow 0} \frac{f_{2}(\varrho)}{f_{1}(\varrho)}=0 .
$$

Proceeding in this way we construct operators $P_{k}(x, \eta \varrho), C_{k}(x, \eta)$ and functions $f_{k}(\varrho)$ by the recursion formulae

$$
\begin{gathered}
C_{k}(x \eta)=\lim _{\varrho \rightarrow 0} \frac{P_{k}(x \eta \varrho)}{f_{k}(\varrho)}, \\
P_{k}(x \eta \varrho)=f_{k}(\varrho) C_{k}(x \eta)+P_{k+1}(x \eta \varrho),
\end{gathered}
$$

where $f_{k}(\varrho)$ is chosen to be one of the most singular matrix elements of $P_{k}$. We continue this procedure until we arrive at an operator $P_{k+1}$ which vanishes in the limit $\varrho \rightarrow 0$. Then the operator product becomes

$$
P(x \eta \varrho)=f_{k}(\varrho) C_{1}(x \eta)+\cdots+f_{n}(\varrho) C_{n}(x \eta)+P_{n+1}(x \eta \varrho)
$$

with

$$
\lim _{\varrho \rightarrow 0} P_{n+1}(x \eta \varrho)=0 .
$$

(1.14) represents the principal part of the expansion near $\varrho=0$. The functions $f_{k}$ carry the singularities of $P$ and are ordered according to decreasing strength of singularity

$$
\begin{gathered}
\lim _{\varrho \rightarrow 0} \frac{f_{k+1}}{f_{k}}=0, \quad \lim f_{k}(\varrho)=\infty \text { for } k=1, \ldots, n-1, \\
\lim _{\varrho \rightarrow 0} f_{n}(\varrho)=\infty \text { or } \lim _{\varrho \rightarrow 0} f_{n}(\varrho) \neq 0 .
\end{gathered}
$$


The field operators $C_{k}$ are given by

$$
C_{k}=\lim _{\varrho \rightarrow 0} \frac{P-\sum_{l=1}^{k} f_{l} C_{l}}{f_{k}}
$$

and satisfy the causality conditions

$$
\begin{aligned}
{\left[C_{k}(x, \eta), O(y)\right]_{ \pm} } & =0, \\
{\left[C_{k}(x, \eta), C_{l}(y, \zeta)\right]_{ \pm} } & =0
\end{aligned}
$$

with commutators or anticommutators taken appropriately. $O(y)$ denotes a field operator which (anti)commutes with $A_{1}, \ldots, A_{n}$ at spacelike distances.

In deriving the principal part (1.14-15) some assumptions have been used which seem to be plausible but at present cannot be inferred from Wightman's postulates. The precise formulation of the hypothesis needed will be given in Section 2 (Eq. (2.15) and Hypothesis 1). Eq. (2.15) excludes oscillations at $\varrho=0$. For models which violate this hypothesis the following alternatives will be found in Section 5:

(a) $P(x, \eta \varrho)$ has no leading singularities as operator, i.e., to any matrix element of $P$ another one can be found which is more singular at $\varrho=0$.

(b) The remainder $P_{n+1}(x, \eta \varrho)$ has no leading singularity as an operator, for some value of $n$. Then (1.14) holds with

$$
\begin{gathered}
\lim _{\varrho \rightarrow 0} \frac{f_{k+1}}{f_{k}}=0, \quad \lim _{k} f_{k}(\varrho)=\infty \text { for } k=1, \ldots, n, \\
\lim _{\varrho \rightarrow 0} \frac{P_{n+1}}{f_{k}}=0 .
\end{gathered}
$$

In this case (1.14) gives the leading singularity for only some of the matrix elements of $P$.

(c) (1.14) and (1.21) hold for any $n$, but no $P_{n}$ vanishes at $\varrho=0$ with all matrix elements.

In order to obtain the asymptotic expansion (1.4) we construct an infinite sequence of operators $C_{k}$ and functions $f_{k}$ using the recursion formulae (1.12-13). If $P_{k}$ vanishes at $\varrho=0$ we take $f_{k}$ as one of the matrix elements of $P_{k}$ which vanish most weakly for $\varrho \rightarrow 0$. Under suitable assumptions on the matrix elements of $P$ (Hypothesis 2 of Section 2) it will be shown in Section 4 that $P_{k}$ and $f_{k}$ vanish stronger than any power of $\varrho$ provided $k$ is sufficiently large. With this we have

$$
P(x \eta \varrho)=f_{1}(\varrho) C_{1}(x \eta)+\cdots+f_{k}(\varrho) C_{k}(x \eta)+P_{k+1}(x \eta \varrho)
$$

where

$$
\lim _{\varrho \rightarrow 0} \frac{P_{k+1}(x \eta \varrho)}{\varrho^{N}}=0
$$


for any $N$ provided $k>k(N)$. In addition the functions $f_{k}$ satisfy

$$
\lim _{\varrho \rightarrow 0} \frac{f_{k+1}(\varrho)}{f_{k}(\varrho)}=0 \text {. }
$$

The local operators $C_{k}$ are given by (1.18) and can be chosen to be linearly independent. The Eq. (1.22-23) represent by definition the asymptotic expansion

$$
P(x \eta \varrho)=\sum_{k=1}^{\infty} f_{k}(\varrho) C_{k}(x \eta) .
$$

The following sections contain the rigorous derivation of these results. In Section 2 the assumptions are formulated from which the principal part (Section 3) and the asymptotic expansion (Section 4) are derived. Alternatives to the assumptions and modifications are discussed in Section 5 .

\section{General Assumptions}

We consider field operators

$$
O_{1}(x), \ldots, O_{c}(x)
$$

which satisfy Wightman's postulates of a local, relativistic quantum field theory [14]. Each of the operators $O_{j}$ may have several components $O_{j \alpha}$ transforming under a representation of the homogeneous Lorentz group. With $O_{j}$ also the hermitian conjugate $O_{j}^{*}$ is listed in (2.1). $D_{0}$ denotes the joint domain of definition of the smeared operators

$$
O_{j}(f)=\int d x f(x) O_{j}(x) \quad f \in \mathscr{S}\left(R_{4}\right),
$$

which is obtained by applying a polynomial of the $O_{j}(f)$ to the vacuum state $\Omega . D_{0}$ is dense in the Hilbert space $\mathscr{H}$.

In the work that follows a family

$$
Q(g)=\int d z_{1} \ldots d z_{n} g\left(z_{1} \ldots z_{n}\right) Q\left(z_{1} \ldots z_{n}\right)
$$

of operators in Hilbert space is called a distribution in $\mathscr{S}_{z_{1} \ldots z_{n}}^{\prime}(\Delta)$

$$
Q\left(z_{1} \ldots z_{n}\right) \in \mathscr{S}_{z_{1} \ldots z_{n}}^{\prime}(\Delta)
$$

if $Q(g)$ is defined for every $g \in \mathscr{S}_{z_{1} \ldots z_{n}}$ on the dense set $\Delta$ and if each matrix element

$$
(\Phi, Q(g) \Psi) \quad \Phi, \Psi \in \Delta
$$

is a distribution in $\mathscr{S}_{z_{1} \ldots z_{n}}^{\prime}(\Delta)$. We further write

if

$$
\lim _{\varrho \rightarrow 0} Q_{\varrho}\left(z_{1} \ldots z_{n}\right)=Q\left(z_{1} \ldots z_{n}\right) \text { in } \mathscr{S}_{z_{1} \ldots z_{n}}^{\prime}(\Delta)
$$

$$
Q \in \mathscr{S}_{z_{1} \ldots z_{n}}^{\prime}(\Delta), \quad Q_{\varrho} \in \mathscr{S}_{z_{1} \ldots z_{n}}^{\prime}(\Delta)
$$


and

$$
\lim _{\varrho \rightarrow 0}\left(\Phi, Q_{\varrho}(g) \Psi\right)=(\Phi, Q(g) \Psi) \quad \text { for } \quad \Phi \in \mathscr{H}, \quad \Psi \in \Delta
$$

(2.4) implies

$$
\lim _{\varrho \rightarrow 0}\left(\Phi, Q_{\varrho}\left(z_{1} \ldots z_{n}\right) \Psi\right)=\left(\Phi, Q\left(z_{1} \ldots z_{n}\right) \Psi\right) \quad \text { in } \quad \mathscr{S}_{z_{1} \ldots z_{n}}^{\prime} \quad \text { if } \quad \Phi, \Psi \in \Delta .
$$

Instead of the operator product (1.2) we introduce the smeared operator

$$
\begin{aligned}
P(t, \varrho) & =\int d x d \eta t(x \eta) P(x \eta \varrho) \\
d \eta & =d \eta_{1} \ldots d \eta_{a-1} \\
P(x \eta \varrho) & =A_{1}\left(x_{1}\right) \ldots A_{a}\left(x_{a}\right)
\end{aligned}
$$

with the variables (1.5). $A_{1}, \ldots, A_{n}$ denote linear combinations of operators $O_{j}$ or derivatives thereof. Our aim is to study the behavior of $P(t, \varrho)$ for $\varrho \rightarrow 0$. First we show that for every real $\varrho \neq 0 P(t, \varrho)$ can be defined as a distribution in $\mathscr{S}_{x \eta}^{\prime}\left(D_{0}\right)$. For $\Phi, \Psi \in D_{0}$ the matrix elements

$\left(\Phi, A_{1}\left(f_{1}\right) \ldots A_{a}\left(f_{a}\right) \Psi\right)=\int d x_{1} \ldots d x_{a} f_{1}\left(x_{1}\right) \ldots f_{a}\left(x_{a}\right)\left(\Phi, A_{1}\left(x_{1}\right) \ldots A_{a}\left(x_{a}\right) \Psi\right)$

may be extended to distributions in the variables $x_{1}, \ldots, x_{a}$. We introduce new independent variables $x, \eta_{1}, \ldots, \eta_{a-1}$ by (1.5). Then

$$
(\Phi, P(t \varrho) \Psi)=\int d x d \eta_{1} \ldots d \eta_{a-1} t\left(x \eta_{1} \ldots \eta_{a-1}\right)(\Phi, P(x \eta \varrho) \Psi)
$$

is a distribution in the variables $x, \eta_{1}, \ldots, \eta_{a-1}$ for $\varrho \neq 0$. Likewise the vacuum expectation values

$$
\left\langle Q_{1} \ldots Q_{n}\right\rangle
$$

with either

$$
Q_{\kappa}=O_{j \kappa}\left(x_{\kappa}\right)
$$

or

$$
Q_{\kappa}=P\left(x_{\kappa} \eta^{(\kappa)} \varrho\right) \quad(\varrho \neq 0)
$$

may be interpreted as distributions in all the variables $x_{\kappa}, \eta_{\kappa_{1}}^{(\kappa)} \ldots \eta_{\kappa_{a}-1}^{(\kappa)}$. From the vacuum expectation values (2.8) the operator (2.5) is constructed on the domain $D_{0}$ in the usual manner. The distributions (2.7) are the matrix elements of $P(t, \varrho)$ between vectors $D_{0}$. Hence $(2.5)$ is a distribution in $\mathscr{S}_{x \eta}^{\prime}\left(D_{0}\right)$.

We state the following properties of $P$.

(i) Lorentz Invariance. Under an inhomogeneous Lorentz transformation

$$
\begin{gathered}
x^{\prime}=\Lambda x+a, \quad \eta_{r}^{\prime}=\Lambda \eta_{r}, \quad \Phi^{\prime}=U(\Lambda, a) \Phi, \quad \Psi^{\prime}=U(\Lambda, a) \Psi \\
t^{\prime}\left(x^{\prime}, \eta_{1}^{\prime}, \ldots, \eta_{a-1}^{\prime}\right)=t\left(x, \eta_{1}, \ldots, \eta_{a-1}\right)
\end{gathered}
$$


the components of $P_{\alpha}$ transform according to

$$
\left(\Phi^{\prime}, P_{\alpha^{\prime}}\left(t^{\prime}, \varrho\right) \Psi^{\prime}\right)=\sum_{\alpha} D_{\alpha^{\prime} \alpha}(\Lambda)\left(\Phi, P_{\alpha}(t, \varrho) \Psi\right) \quad \Phi \in H, \quad \Psi \in D_{0}
$$

where $D(\Lambda)$ is a finite dimensional representation of the homogeneous Lorentz group.

(ii) Causality. Let $t \in \mathscr{D}_{x \eta}, f \in \mathscr{D}_{\eta}$ be test functions satisfying

$$
t\left(x \eta_{1} \ldots \eta_{a-1}\right) f(\eta)=0
$$

if $\left(x_{r}-y\right)^{2} \geqq 0$ for at least one $r=1, \ldots, n$. Then

$$
\left[P(t, \varrho), O_{j}(f)\right]_{ \pm} \Psi=0 \quad \text { if } \quad \Psi \in D_{0} .
$$

We want to formulate a hypothesis concerning the relative growth of matrix elements of $P$ for $\varrho \rightarrow 0$. To this end we consider the set $\mathscr{A}_{N}$ of all functions

$$
\phi(\varrho)=\sum_{\kappa=1}^{A}\left(\Phi_{\kappa}, P_{\alpha_{\kappa}}\left(t_{\kappa}, \varrho\right) \Psi_{\kappa}\right), \quad \Psi_{\kappa} \in D_{0}
$$

which do not vanish stronger than $\varrho^{N}$ in the limit $\varrho \rightarrow 0$, i.e. ${ }^{4}$

$$
\lim _{\varrho \rightarrow 0} \frac{\phi(\varrho)}{\varrho^{N}} \neq 0 \text {. }
$$

In (2.13) the $\Phi_{\kappa}$ are arbitrary vectors of Hilbert space, the $\Psi_{\kappa}$ are elements of the domain $D_{0}$. The $t_{\kappa}$ denote test functions in $\mathscr{S}\left(R_{4 a}\right)$.

We first assume that the ratio of any given two functions of $\mathscr{A}_{N}$ has only one accumulation point, i.e.,

$$
\lim _{\varrho \rightarrow 0} \frac{\phi_{1}(\varrho)}{\phi_{2}(\varrho)} \text { exists or } \lim _{\varrho \rightarrow 0} \frac{\phi_{1}(\varrho)}{\phi_{2}(\varrho)}=\infty \text {. }
$$

We then define an equivalence relation in $\mathscr{A}_{N} \cdot \phi_{1}, \phi_{2}$ are said to have the same behavior near $\varrho=0$

$$
\phi_{1} \sim \phi_{2}
$$

if their ratio is finite in the limit $\varrho \rightarrow 0$

$$
\lim _{\varrho \rightarrow 0} \frac{\phi_{1}}{\phi_{2}} \neq 0, \infty \text {. }
$$

Otherwise we say that $\phi_{1}$ and $\phi_{2}$ behave differently near $\varrho=0$ and use the notation

$$
\phi_{1}>\phi_{2} \quad \text { if } \lim \frac{\phi_{1}}{\phi_{2}}=\infty
$$

${ }^{4}$ This includes the case that $\lim _{\varrho \rightarrow 0} \frac{\phi(\varrho)}{\varrho^{N}}=\infty$. 
and

$$
\phi_{1}<\phi_{2} \quad \text { if } \lim \frac{\phi_{1}}{\phi_{2}}=0
$$

With respect to the relation $\sim$ we introduce equivalence classes of $\mathscr{A}_{N}$. For two equivalence classes $\mathscr{C}, \mathscr{C}^{\prime}$ we write

if

$$
\mathscr{C}>\mathscr{C}^{\prime}
$$

$$
\lim _{\varrho \rightarrow 0} \frac{\phi}{\phi^{\prime}}=\infty \quad \text { for } \quad \phi \in \mathscr{C}, \quad \phi^{\prime} \in \mathscr{C}^{\prime}
$$

We formulate the following hypotheses:

Hypothesis 1. There are at most $n$ functions (2.13) which do not vanish at $\varrho=0$ and behave differently near $\varrho=0$. In other words, the number of equivalence classes of $\mathscr{A}_{0}$ is finite.

Hypothesis 2. For every $N$ there are at most $n(N)$ functions (2.13) which do not vanish stronger than $\varrho^{N}$ and behave differently near $\varrho=0$. In other words, the number of equivalence classes of $\mathscr{A}_{N}$ is finite.

Hypothesis 3. $\mathscr{A}$ denotes the set of all functions (2.13) without further restrictions. (2.15) is again assumed for any two functions of $\mathscr{A}$.

Let $\phi$ be a function of $\mathscr{A}$ and $\mathscr{A}_{\varphi}$ denote the set of all $\chi \in \mathscr{A}$ with $\chi<\phi$. Then we assume that $\mathscr{A}$ and any $\mathscr{A}_{\varphi}$ contain maximal elements.

An element $\psi$ of a subset $\mathscr{B} \subseteq \mathscr{A}$ is called maximal if

for any other $\chi \in \mathscr{B}$.

$$
\psi>\chi \quad \text { or } \quad \psi \sim \chi
$$

\section{Principal Part}

The operator $P_{\alpha}(t, \varrho)$ was defined by Eq. (2.5) as a distribution in $\mathscr{S}_{x \eta}^{\prime}\left(D_{0}\right)$. We prove for $P_{\alpha}$ the following

Theorem 1. Hypothesis 1 is necessary and sufficient for the existence of the principal part

$$
P_{\alpha}(t, \varrho)=\sum_{k=1}^{n} f_{k}(\varrho) C_{k \alpha}(t)+R_{\alpha}(t, \varrho)
$$

with the properties

(a) The remainder $R$ is a distribution in $\mathscr{S}_{x \eta}^{\prime}\left(D_{0}\right)$ and vanishes weakly for $\varrho \rightarrow 0$

$$
\lim _{\varrho \rightarrow 0}(\Phi, R(t, \varrho) \Psi)=0 \quad \text { for } \quad \Phi \in \mathscr{H}, \quad \Psi \in D_{0}
$$


(b) Near $\varrho=0$ the functions $f_{k}(\varrho)$ are non-vanishing and ordered according to decreasing singularity

$$
\begin{gathered}
\lim _{\varrho \rightarrow 0} \frac{f_{k+1}(\varrho)}{f_{k}(\varrho)}=0 . \\
\lim _{\varrho \rightarrow 0} f_{n}(\varrho) \neq 0 .
\end{gathered}
$$

(c) The $C_{k}(t)$ are linear operators on $D_{0}$ and distributions in $\mathscr{S}_{x \eta}^{\prime}\left(D_{0}\right)$. The operators $C_{k}(t)$ can be chosen linearly independent. The Lorentz invariance of the theory implies

Lorentz Invariance of $C_{\boldsymbol{k}}(t)$. Under a homogeneous Lorentz transformation (2.9) the components $C_{k \alpha}$ transform according to

$$
\left(\Phi^{\prime}, C_{k \alpha^{\prime}}\left(t^{\prime}\right) \Psi^{\prime}\right)=\sum_{\alpha} D_{\alpha^{\prime} \alpha}(\Lambda)\left(\Phi, C_{k \alpha}(t) \Psi\right) \quad \Phi \in \mathscr{H}, \quad \Psi \in D_{0} .
$$

The causality postulate implies

Locality of $C_{\boldsymbol{k}}(t)$. The operators $C_{k}(t)$ and $O_{j}(f)$ (anti-) commute

$$
\left[C_{k}(t), O_{j}(r)\right]_{ \pm} \Psi=0 \quad \text { for } \quad \Psi \in D_{0}
$$

if the test functions $t \in \mathscr{D}_{x \eta}$ and $f \in \mathscr{D}_{y}$ satisfy

$$
t\left(x \eta_{1} \ldots \eta_{a-1}\right) f(y)=0 \quad \text { for } \quad(x-y)^{2} \geqq 0 .
$$

The operators $C_{k}(t)$ and $C_{l}(s)$ (anti-) commute

$$
\left(C_{k}^{*}(t) \Phi, C_{l}(s) \Psi\right) \pm\left(C_{l}^{*}(s) \Phi, C_{k}(t) \Psi\right)=0 \quad \text { for } \quad \Phi, \Psi \in D_{0}
$$

if the test functions satisfy

$$
t\left(x \eta_{1} \ldots \eta_{a-1}\right) s\left(y \zeta_{1} \ldots \zeta_{a-1}\right)=0 \text { for }(x-y)^{2} \geqq 0 .
$$

Proof. We first show that Hypothesis 1 follows from (3.1) and (a)-(c). Let $\Phi \in \mathscr{H}, \Psi \in D_{0}$, then each matrix element of $P_{\alpha}$ is represented by

$$
\begin{aligned}
\left(\Phi, P_{\alpha}(t, \varrho) \Psi\right)= & \sum_{k=1}^{n} f_{k}(\varrho)\left(\Phi, C_{k \alpha}(t) \Psi\right)+\left(\Phi, R_{\alpha}(t, \varrho) \Psi\right) \\
& \lim _{\varrho \rightarrow 0}\left(\Phi, R_{\alpha}(t, \varrho) \Psi\right)=0
\end{aligned}
$$

which shows that $\mathscr{A}_{0}$ has only a finite number of equivalence classes.

We next prove that Hypothesis 1 is sufficient. If $\mathscr{A}_{0}$ is empty

$$
\lim _{\varrho \rightarrow 0}\left(\Phi, P_{\alpha}(t, \varrho) \Psi\right)=0, \quad \Psi \in D_{0}
$$

and we may set

$$
P_{\alpha}(t, \varrho)=R_{\alpha}(t, \varrho)
$$


If $\mathscr{A}_{0}$ is not empty we denote its equivalence classes by $\mathscr{C}_{1}, \ldots, \mathscr{C}_{n}$ and number them according to decreasing strength of singularities

$$
\mathscr{C}_{1}>\cdots>\mathscr{C}_{n} \text {. }
$$

Let

$$
\phi(\varrho)=\Sigma\left(\Phi_{\kappa}, P_{\alpha_{\kappa}}\left(t_{\kappa}, \varrho\right) \Psi_{\kappa}\right) \in \mathscr{C}_{1}
$$

be an element of $\mathscr{C}_{1}$. Since either

$$
\lim _{\varrho \rightarrow 0}\left(\Phi_{\kappa}, P_{\alpha_{\kappa}}\left(t_{\kappa}, \varrho\right) \Psi_{\kappa}\right)=0
$$

or

$$
\left(\Phi_{\kappa}, P_{\alpha_{\kappa}}\left(t_{\kappa}, \varrho\right) \Psi_{\kappa}\right) \in \mathscr{C}_{j(\kappa)} \leqq \mathscr{C}_{1}
$$

there must be a matrix element

$$
f_{1}(\varrho)=\left(\tilde{\Phi}, P_{\tilde{\alpha}}(\tilde{t}, \varrho) \tilde{\Psi}\right) \in \mathscr{C}_{1} \quad \tilde{\Phi}=\Phi_{\kappa}, \quad \tilde{\Psi}=\Psi_{\kappa}, \quad t=\tilde{t}_{\kappa}, \quad \tilde{\alpha}=\alpha_{\kappa}
$$

belonging to $\mathscr{C}_{1}$ for at least one value of $\kappa$. We will show that the limit

$$
C_{1}(t)=\lim _{\varrho \rightarrow 0} \frac{P(t, \varrho)}{f_{1}(\varrho)}
$$

exists in the weak sense and defines a non-vanishing field operator. First we note that the limit

$$
\lim _{\varrho \rightarrow 0} \frac{(\Phi, P(t, \varrho) \Psi)}{f_{1}(\varrho)}
$$

exists for all $\Phi \in \mathscr{H}, \Psi \in D_{0}$. It does not vanish identically since

$$
\lim _{\varrho \rightarrow 0} \frac{\left(\tilde{\Phi}, P_{\tilde{\alpha}}(\tilde{t}, \varrho) \tilde{\Psi}\right)}{f_{1}(\varrho)}=1
$$

(3.13) implies the existence of a vector $X(t)$ with

$$
\lim _{\varrho \rightarrow 0}\left(\Phi, \frac{P(t, \varrho)}{f_{1}(\varrho)} \Psi\right)=(\Phi, X(t))
$$

for every $\Phi \in \mathscr{H}, \Psi \in D_{0}{ }^{5}$.

$$
X=C_{1}(t) \Psi
$$

defines a linear operator $C_{1}(t)$ on the domain $D_{0}$. This yields (3.12) in the sense of weak convergence.

Since

$$
\left(\Phi, C_{1}(t) \Psi\right)=\lim _{\varrho \rightarrow 0} \frac{(\Phi, P(t, \varrho) \Psi)}{f_{1}(\varrho)}
$$

\footnotetext{
${ }^{5}$ This follows from the weak completeness of the Hilbert space.
} 
the matrix elements $\left(\Phi, C_{1}(t) \Psi\right)$ are distributions in $\mathscr{S}_{x \eta}^{\prime}$ for every $\Phi, \Psi \in D_{0}$. Hence

$$
C_{1}(x \eta)=\lim _{\varrho \rightarrow 0} \frac{P(t \eta \varrho)}{f_{1}(\varrho)}
$$

exists as a distribution in $\mathscr{S}_{x \eta}^{\prime}\left(D_{0}\right)$.

Starting from

$$
P_{1}(t, \varrho)=P(t, \varrho), \quad C_{1}(t) \quad \text { and } \quad f_{1}(\varrho)
$$

we will construct a sequence of operators $P_{k}(t, \varrho), C_{k}(t)$ and functions $f_{k}(\varrho)$ which are related by the recursion formulae

$$
\begin{gathered}
C_{k}(t)=\lim _{\varrho \rightarrow 0} \frac{P_{k}(t, \varrho)}{f_{k}(\varrho)} \neq 0, \\
P_{k}(t, \varrho)=f_{k}(\varrho) C_{k}(t)+P_{k+1}(t, \varrho) .
\end{gathered}
$$

We use the following hypothesis of induction:

(i) Distribution properties. For $\varrho \neq 0$ the operator $P_{k}(t, \varrho)$ is a distribution in $\mathscr{S}_{x \eta}^{\prime}\left(D_{0}\right)$.

(ii) Growth of matrix elements for $\varrho \rightarrow 0$. Let $\mathscr{A}_{0}\left(P_{k}\right)$ denote the set of all functions

$\phi(\varrho)=\Sigma\left(\Phi_{\kappa}, P_{k \alpha_{\kappa}}\left(t_{\kappa}, \varrho\right) \Psi_{\kappa}\right), \quad \Phi_{\kappa} \in \mathscr{H}, \quad \Psi_{\kappa} \in D_{0}, \quad t_{\kappa} \in \mathscr{S}\left(R_{4 a}\right)$

with the property

$$
\lim _{\varrho \rightarrow 0} \phi(\varrho) \neq 0 .
$$

Then

$$
\mathscr{A}_{0}\left(P_{k}\right)=\sum_{j=k}^{n} \mathscr{C}_{j}
$$

Under this hypothesis we will prove that (3.17) exists and that the operator $P_{k+1}$ defined by (3.18) again satisfies the conditions of the hypothesis.

First we choose the function $f_{k}(\varrho)$ which will be used for defining $C_{k}$ as a non-vanishing operator. According to statement (ii) of the hypothesis any function $\phi \in \mathscr{C}_{k}$ is also an element of $\mathscr{A}_{0}\left(P_{k}\right)$ and can therefore be written as

Since either

$$
\phi(\varrho)=\Sigma\left(\Phi_{\kappa}, P_{k \alpha_{\kappa}}\left(t_{\kappa}, \varrho\right) \Psi_{\kappa}\right), \quad \Psi_{\kappa} \in D_{0} .
$$

$$
\lim _{\varrho \rightarrow 0}\left(\Phi_{\kappa}, P_{k \alpha_{\kappa}}\left(t_{\kappa}, \varrho\right) \Psi_{\kappa}\right)=0
$$

or

$$
\left(\Phi_{\kappa}, P_{k \alpha_{\kappa}}\left(t_{\kappa}, \varrho\right) \Psi_{\kappa}\right) \in \mathscr{C}_{j(\kappa)} \leqq \mathscr{C}_{k}
$$


it follows

$$
\left(\Phi_{\kappa}, P_{k \alpha_{\kappa}}\left(t_{\kappa}, \varrho\right) \Psi_{\kappa}\right) \in \mathscr{C}_{k}
$$

for at least one value of $\kappa$. We choose (3.22) as the function $f_{k}(\varrho)$. As in the case of $C_{1}$ it follows that the weak limit (3.17) defines a non-vanishing distribution in $\mathscr{S}_{x \eta}^{\prime}\left(D_{0}\right)$. By (3.18) the distribution properties (i) follow for $P_{k+1}$.

In order to prove (3.21) for $P_{k+1}$ we first show

$$
\mathscr{A}_{0}\left(P_{k+1}\right) \subseteq \mathscr{A}_{0}\left(P_{k}\right) \text {. }
$$

Indeed we have

$$
\Sigma\left(\Phi_{\kappa}, P_{k+1, \alpha_{\kappa}}\left(t_{\kappa}, \varrho\right) \Psi_{\kappa}\right)=\Sigma\left(\hat{\Phi}_{\kappa}, P_{k \tilde{\alpha}_{\kappa}}\left(\hat{t}_{\kappa}, \varrho\right) \hat{\Psi}_{\kappa}\right) \in \mathscr{A}_{0}\left(P_{k}\right)
$$

since

$$
\begin{gathered}
\left(\Phi, P_{k+1, \alpha}(t, \varrho) \Psi\right)=\left(\Phi, P_{k, \alpha}(t, \varrho) \Psi\right)-\left(\tilde{\Phi}, P_{k, \tilde{\alpha}}(\tilde{t}, \varrho) X\right) \\
X=\left(\Phi, C_{k, \alpha}(t) \Psi\right) \tilde{\Psi}, \quad f_{k}(\varrho)=\left(\tilde{\Phi}, P_{k, \tilde{\alpha}}(\tilde{t}, \varrho) \tilde{\Psi}\right) .
\end{gathered}
$$

Next we show that the type $\mathscr{C}_{k}$ is missing in $\mathscr{A}_{0}\left(P_{k+1}\right)$. Since

$$
\lim _{\varrho \rightarrow 0} \frac{\left(\Phi, P_{k+1}(t, \varrho) \Psi\right)}{f_{k}(\varrho)}=\lim _{\varrho \rightarrow 0} \frac{\left(\Phi, C_{k}(t, \varrho) \Psi\right)}{f_{k}(\varrho)}-\left(\Phi, C_{k}(t) \Psi\right)=0
$$

holds for any matrix element of $P_{k+1}$ between $\Phi \in \mathscr{H}, \Psi \in D_{0}$ it follows

$$
\lim _{\varrho \rightarrow 0} \frac{\phi(\varrho)}{f_{k}(\varrho)}=0
$$

for $\phi(\varrho) \in \mathscr{A}_{0}\left(P_{k+1}\right)$. Hence $\phi(\varrho)$ belongs to a type $\mathscr{C}$ with $\mathscr{C}<\mathscr{C}_{k}$

$$
\mathscr{C}_{k} \cap \mathscr{A}_{0}\left(P_{k+1}\right)=\emptyset \text {. }
$$

Finally we check that any $\mathscr{C}_{l}(l>j)$ is contained in $\mathscr{A}_{0}\left(P_{k+1}\right)$. Let $\phi \in \mathscr{C}_{l}(l>k)$, then (statement (ii) for $P_{k}$ )

$$
\begin{aligned}
\phi(\varrho)= & \Sigma\left(\Phi_{\kappa}, P_{k \alpha_{\kappa}}\left(t_{\kappa}, \varrho\right) \Psi_{\kappa}\right)=f_{k}(\varrho) \Sigma\left(\Phi_{\kappa}, C_{k \alpha_{\kappa}}\left(t_{\kappa}\right) \Psi_{\kappa}\right) \\
& +\Sigma\left(\Phi_{\kappa}, P_{k+1, \alpha_{\kappa}}\left(t_{\kappa}, \varrho\right) \Psi_{\kappa}\right) .
\end{aligned}
$$

From $f_{k} \in \mathscr{C}_{k}$ and

$$
\left(\Phi_{\kappa}, P_{k+1, \alpha_{\kappa}}\left(t_{\kappa}, \varrho\right) \Psi_{\kappa}\right)<f_{k}
$$

it follows that

$$
\Sigma\left(\Phi_{\kappa}, C_{k \alpha_{\kappa}}\left(t_{\kappa}\right) \Psi_{\kappa}\right)=0
$$

and

Hence

$$
\phi(\varrho)=\Sigma\left(\Phi_{\kappa}, P_{k+1, \alpha_{\kappa}}\left(t_{\kappa}, \varrho\right) \Psi_{\kappa}\right) .
$$

$$
\mathscr{C}_{l} \subseteq \mathscr{A}_{0}\left(P_{k+1}\right) \text { for } \quad l>k
$$


(3.23-25) imply the desired relation

$$
\mathscr{A}_{0}\left(P_{k+1}\right)=\sum_{\alpha=k+1}^{n} \mathscr{C}_{k} .
$$

This completes the proof of the hypothesis of induction.

Iterating the recursion formula (3.18) we obtain (3.1) with

$$
R(t, \varrho)=P_{n+1}(t, \varrho) .
$$

Since $A_{0}\left(P_{n+1}\right)=\emptyset$ we have (3.2). $f_{k}(\varrho) \in \mathscr{C}_{k}$ implies (3.3-4). Hence all conditions (a)-(c) are fulfilled.

In order to check the transformation law (3.5) we assume that for some value of $k$ the relation

$\left(\Phi^{\prime}, P_{k \alpha^{\prime}}\left(t^{\prime}, \varrho\right) \Psi^{\prime}\right)=\sum_{\alpha} D_{\alpha^{\prime} \alpha}(\Lambda)\left(\Phi, P_{k \alpha}(t, \varrho) \Psi\right) \quad \Phi \in \mathscr{H}, \quad \Psi \in D_{0}$

has been shown for a Lorentz transformation (2.9). Dividing (3.26) by $f_{k}(\varrho)$ and taking the limit $\varrho \rightarrow 0$ we obtain

$$
\left(\Phi^{\prime}, C_{k \alpha^{\prime}}\left(t^{\prime}\right) \Psi^{\prime}\right)=\sum_{\alpha} D_{\alpha^{\prime} \alpha}(\Lambda)\left(\Phi, C_{k \alpha}(t) \Psi\right) \quad \Phi \in \mathscr{H}, \quad \Psi \in D_{0}
$$

as transformation law for $C_{k}$. By the recursion formula (3.18) relation (3.26) is then also true for $P_{k+1}$.

For the proof of the local properties we assume as hypothesis of induction that

(i) the (anti)commutation relation

$$
\left[P_{k}(t, \varrho), O_{j}(f)\right]_{ \pm} \Psi=0, \quad \Psi \in D_{0}
$$

holds for test function $t \in \mathscr{S}_{x \eta}, f \in \mathscr{S}_{y}$ which satisfy

$$
t\left(x, \eta_{1} \ldots \eta_{a-1}\right) f(y)=0
$$

unless

$(x-y)^{2}<0 \quad$ and $\quad\left(x_{r}-y\right)^{2}<0, \quad r=1, \ldots, a, \quad x_{r}=x+\varrho \lambda_{r}(\eta)$.

(ii) The (anti)commutation relation

$$
\begin{gathered}
\left(P_{k}(t, \varrho)^{*} \Phi, C_{j}(s) \Psi\right) \pm\left(C_{j}^{*}(s) \Phi, P_{k}(t, \varrho) \Psi\right)=0 \\
\quad \text { for } \Phi, \Psi \in D_{0}, \quad j=1, \ldots, k-1
\end{gathered}
$$

holds for test functions $t \in \mathscr{D}_{\alpha \eta}, s \in \mathscr{D}_{v \zeta}$ which satisfy

$$
t\left(x \eta_{1} \ldots \eta_{a-1}\right) s\left(y \zeta_{1} \ldots \zeta_{a-1}\right)=0
$$

unless (3.30) is valid. 
(iii) The (anti)commutation relation

$$
\begin{gathered}
\left(P_{k}(t, \varrho)^{*} \Phi, P_{k}(s, \sigma) \Psi\right) \pm\left(P_{k}(s, \sigma)^{*} \Phi, P_{k}(t, \varrho) \Psi\right)=0 \\
\text { for } \Phi, \Psi \in D_{0}
\end{gathered}
$$

holds for test functions $t \in \mathscr{D}_{x \eta_{1}}, s \in \mathscr{D}_{y \zeta}$ which satisfy (3.32) unless

$$
\begin{gathered}
(x-y)^{2}<0 \quad \text { and } \quad\left(x_{r}-y_{n}\right)^{2}<0 \\
x_{r}=x+\varrho \lambda_{r}(\eta), \quad y_{n}=y+\sigma \lambda_{r}(\zeta) .
\end{gathered}
$$

Under this hypothesis we will prove that the operator $P_{k+1}$ again satisfies the conditions (i)-(iii).

Suppose the test function $t \in \mathscr{D}_{x \eta}$ and $f \in \mathscr{D}_{\underline{y}}$ satisfy

$$
t\left(x \eta_{1} \ldots \eta_{a-1}\right) f(y)=0 \quad \text { if }(x-y)^{2} \geqq 0 .
$$

Then (3.28) holds provided $\varrho$ is small enough that all $x+\varrho \eta_{r}$ in the support of $t$ are sufficiently close to $x$ such that (3.29-30) is satisfied. For matrix elements between vectors $\Phi, \Psi \in D_{0}$ one obtains

$$
\begin{gathered}
0=\left(\Phi,\left[P(t, \varrho), O_{j}(t)\right]_{ \pm} \Psi\right)=\left(\Phi, P(t, \varrho) \Psi^{\prime}\right) \pm\left(\Phi^{\prime}, P(t, \varrho) \Psi\right) \\
\Psi^{\prime}=O_{j}(f) \Psi, \quad \Phi^{\prime}=O_{j}^{*}(f) \Phi .
\end{gathered}
$$

Dividing by $f_{k}(\varrho)$ and taking the limit $\varrho \rightarrow 0$

$$
0=\left(\Phi, C_{k}(t) \Psi^{\prime}\right) \pm\left(\Phi^{\prime}, C_{k}(t) \Psi\right)=\left(\Phi,\left[C_{k}(t), O_{j}(f)\right]_{ \pm} \Psi\right)
$$

follows. Since this relation holds for any $\Phi \in D_{0}$ we have

$$
\left[C_{k}(t), O_{j}(f)\right]_{ \pm} \Psi=0 \quad \Psi \in D_{0}
$$

for test functions satisfying (3.35). (3.28) and (3.36) imply

$$
\left[P_{k+1}(t, \varrho), O_{j}(f)\right]_{ \pm} \Psi=0 \quad \Psi \in D_{0}
$$

for test functions which satisfy (3.29) unless (3.30) is valid.

Dividing (3.33) by $f_{k}(\sigma)$ and taking the limit $\sigma \rightarrow 0$ we find that (3.31) is also valid for $j=k^{6}$. Dividing (3.31) by $f_{k}(\varrho)$ and taking the limit $\varrho \rightarrow 0$ we obtain

$$
\begin{gathered}
\left(C_{k}(t)^{*} \Phi, C_{j}(s) \Psi\right) \pm\left(C_{j}^{*}(s) \Phi, C_{k}(t) \Psi\right)=0 \\
\text { for } \quad \Phi, \Psi \in D_{0}, \quad j=1, \ldots, k
\end{gathered}
$$

${ }^{6}$ It is used here that the adjoint operators $C_{k}^{*}(t), P_{k}^{*}(t \varrho)$ are defined on $D_{0}$ and satisfy

$$
C_{k}^{*}(t)=\lim _{\varrho \rightarrow 0} \frac{P_{k}^{*}(t \varrho)}{F_{k}(\varrho)}
$$

weakly on $D_{0}$. A proof of this statement will be given in a separate paper by comparison of the expansions of $P_{1}(t \varrho)$ and $P_{1}^{*}(t \varrho)$. 
for test functions satisfying

$$
t\left(x \eta_{1} \ldots \eta_{a-1}\right) s\left(y \zeta_{1} \ldots \zeta_{a-1}\right)=0 \quad \text { if } \quad(x-y)^{2} \geqq 0 .
$$

From (3.31) and (3.38)

$$
\begin{gathered}
\left(P_{k+1}(t)^{*} \Phi, C_{j}(s) \Psi\right) \pm\left(C_{j}(s)^{*} \Phi, P_{k+1}(t) \Psi\right)=0, \\
\Phi, \Psi \in D_{0}, \quad j=1, \ldots, k,
\end{gathered}
$$

follows for test functions which satisfy (3.29) unless (3.30) holds.

For test functions $t, s$ with (3.32) unless (3.34) the relations

$$
\begin{gathered}
\left(P_{k+1}(t, \varrho)^{*} \Phi, P_{k+1}(s, \sigma) \Psi\right) \pm\left(P_{k+1}(s, \sigma)^{*} \Phi, P_{k+1}(t, \varrho) \Psi\right)=0 \\
\text { for } \Phi, \Psi \in D_{0}
\end{gathered}
$$

follow from (3.33), (3.31) (with $j=k$ ) and (3.38).

The relations (3.37), (3.40-41) confirm the local properties (i)-(iii) for the operator $P_{k+1}$. (3.36) and (3.38) represent the local properties (3.6-9) stated in Theorem 1.

We finally prove that the $C_{k}$ can be chosen to be linearly independent without changing the properties (3.1-9). Suppose there is a linear relation

$$
\sum_{\kappa=1}^{n} a_{\kappa} C_{k_{\kappa}}=0
$$

with all $a_{\kappa} \neq 0$. We express the $C_{k_{\kappa}}$ with the highest subscript $b=k_{\kappa}$ by $C_{1}, \ldots, C_{b-1}$

Then

$$
C_{b}=-\sum_{k=1}^{b-1} \beta_{k} C_{k}
$$

$$
P=\sum_{k=1}^{b-1}\left(f_{k}+\beta_{k} f_{b}\right) C_{k}+\sum_{j=1}^{n} f_{k} C_{k}+P_{n+1} .
$$

The new coefficients again satisfy the conditions (3.3-4). By induction all linear relations among the $C_{k}$ can be eliminated until an expansion (3.1) with linearly independent $C_{k}$ is obtained. This completes the proof of Theorem 1.

\section{Asymptotic Expansion}

Concerning the asymptotic expansion of $P(t, \varrho)$ near $\varrho=0$ we state the following

Theorem 2. Hypothesis 2 is necessary and sufficient for the asymptotic expansion

$$
P(t, \varrho)=f_{1}(\varrho) C_{1}(t)+\cdots+f_{k}(\varrho)\left(k(t)+P_{n+1}(t, \varrho)\right)
$$


with the properties

(a) the remainder $P_{k+1}$ is a distribution in $\mathscr{S}_{x \eta}^{\prime}\left(D_{0}\right)$ and satisfies

$$
\begin{aligned}
& \lim _{\varrho \rightarrow 0} \frac{\left(\Phi, P_{k+1}(t, \varrho) \Psi\right)}{\varrho^{N}}=0 \text { if } k>k(N), \\
& \lim _{\varrho \rightarrow 0} \frac{\left(\Phi, P_{k+1}(t, \varrho) \Psi\right)}{f_{k}(\varrho)}=0 .
\end{aligned}
$$

(b) the functions $f_{k}$ satisfy

$$
\lim _{\varrho \rightarrow 0} \frac{f_{k+1}(\varrho)}{f_{k}(\varrho)}=0 .
$$

(c) The $C_{k}(t)$ are linear operators on $D_{0}$ and distributions in $\mathscr{S}_{x \eta}^{\prime}\left(D_{0}\right)$. It can be arranged that a finite number of $C_{k}(t)$ are linearly independent. Moreover, the operators $C_{k}(t)$ have the invariance and locality properties stated in Theorem 1.

Proof. Let $\mathscr{B}$ denote the set of all functions (2.13) which satisfy (2.14) for some value of $N$. We have

$$
\mathscr{B}=\sum_{N=1}^{\infty} \mathscr{A}_{N}
$$

We denote the equivalence classes of $\mathscr{B}$ by

$$
\mathscr{C}_{1}>\cdots>\mathscr{C}_{k}>\cdots
$$

where

$$
\mathscr{C}_{1}>\cdots>\mathscr{C}_{n(N)}
$$

are the equivalence classes of $\mathscr{A}_{N}$. The induction procedure of the last section is then easily extended to any value of $k$. To this end statement (ii) (below Eq. (3.18)) is generalized to

(ii) Let $\mathscr{A}_{N}\left(P_{k}\right)$ denote the set of all functions

$$
\begin{gathered}
\phi(\varrho)=\Sigma\left(\Phi_{\kappa}, P_{k \alpha_{\kappa}}\left(t_{\kappa}, \varrho\right) \Psi_{\kappa}\right) \\
\Phi_{\kappa} \in \mathscr{H}, \quad \Psi_{\kappa} \in D_{0}, \quad t_{\kappa} \in \mathscr{S}_{x \eta}
\end{gathered}
$$

with the property

$$
\lim _{\varrho \rightarrow 0} \frac{\phi(\varrho)}{\varrho^{N}} \neq 0
$$

then

$$
\mathscr{A}_{N}\left(P_{k}\right)=\sum_{j=k}^{n(N)} \mathscr{C}_{j}
$$

Eq. (4.2) then follows from the fact that the set $\mathscr{A}_{N}\left(P_{k+1}\right)$ is empty for $k>n(N)$. 
We finally have

Theorem 3. Hypothesis 3 is necessary and sufficient for the expansion (4.1) with properties (a), (b) and (c) except for Eq. (4.2).

For the proof we construct an infinite sequence of equivalence classes

$$
\mathscr{C}_{1}>\mathscr{C}_{2}>\cdots
$$

of $\mathscr{A}$ in the following way. $\mathscr{C}_{1}$ is the set of all maximal elements of $\mathscr{A}$. Let $\phi$ be an element of $\mathscr{C}_{k}$. Then $\mathscr{C}_{k+1}$ is defined as the set of all maximal elements of $\mathscr{A}_{\varphi}$. The induction procedure of the last section can then be applied to the sequence (4.5).

\section{Miscellaneous Remarks}

Throughout this work Eq. (2.15) was assumed which excludes oscillations at $\varrho=0$. If $(2: 15)$ is violated operator product expansions may still be set up by considering special sequences $\varrho_{n}$ with $\lim _{\varrho \rightarrow \infty} \varrho_{n}=0$.

The Hypotheses 1-3 can be relaxed in many ways by restricting the test functions and state vectors in the definition of the functions (2.31). We list the following possibilities:

(1) The test functions $t_{\kappa}$ are different from zero for space-like $\eta$ only

$$
t_{\kappa}(x, \eta)=0 \quad \text { if } \quad \eta_{j}^{2} \geqq 0 .
$$

(2) The test functions $t_{\kappa}$ are different from zero for time-like $\eta$ only

$$
t_{\kappa}(x, \eta)=o \text { if } \eta_{j}^{2} \leqq 0 .
$$

(3) The Fourier transforms of $t_{\kappa}$ have compact support.

(4) The vectors $\Phi_{\kappa}, \Psi_{\kappa}$ represent states of bounded energy-momentum. The definition of classes $\mathscr{A}_{N}$ is then modified accordingly. If Hypotheses 1 or 2 are used with (4) the expressions

$$
\left(\Phi, C_{k}(t) \Psi\right)
$$

might not define linear operators $C_{k}(t)$ in Hilbert space. But (5.1) may still be interpreted as a bilinear form in $\Phi$ and $\Psi$.

We next discuss the alternatives which occur in case Hypothesis 1 is not valid in any acceptable form. Let $\mathscr{A}_{0}$ be the class of functions (2.13) which do not vanish for $\varrho \rightarrow 0$ and are defined with appropriate restrictions on the test functions and state vectors. If Hypothesis 1 does not hold there are an infinite number of equivalence classes in $\mathscr{A}_{0}$. They are totally ordered by the relation $>$. We then have the following cases

(a) There exists no maximal element among the equivalence classes of $\mathscr{A}_{0}$. Then to any function (2.31) which does not vanish at $\varrho=0$ another 
function $\phi^{\prime}$ in $\mathscr{A}_{0}$ can be found such that

$$
\lim _{\varrho \rightarrow 0} \frac{\phi^{\prime}}{\phi}=\infty \text {. }
$$

This means that $P(t, \varrho)$, considered as an operator, does not have a leading singularity.

(b) There exists a maximal element $\mathscr{C}_{1}$ among the equivalence classes of $\mathscr{A}_{0}$. Then a sequence of classes

$$
\mathscr{C}_{1}>\mathscr{C}_{2}>\cdots>\mathscr{C}_{j}>\cdots
$$

can be constructed such that $\Gamma_{j}$ is the maximal element of the difference set

$$
\Delta_{j}=\mathscr{A}_{0}-\bigcup_{\alpha=1}^{j-1} \mathscr{C}_{\alpha} .
$$

We then have the two possibilities

$\left(b_{1}\right)$ The sequence (5.2) terminates for $j=m$ because $\Delta_{j}$ has no maximal element. Then (3.1) holds for $n=m$, but some matrix elements of $R$ diverge for $\varrho \rightarrow 0$ and the operator $R$ does not have a leading singularity. Eq. (3.1) gives the leading singularity for at least some matrix elements of $P$.

$\left(b_{2}\right)$ The sequence (5.2) does not terminate. Then (3.1) holds for any $n$, but some matrix elements of $R$ diverge for $\varrho \rightarrow 0$.

The form of the principal part and the asymptotic series is of course not unique. New operators $C_{k}^{\prime}$ and functions $f_{k}^{\prime}$ may be introduced by certain triangular transformations without changing the conditions (3.2-6) and (4.2-4). These problems will be discussed in a separate paper.

One of us (W.Z.) thanks Dr. G. Dell'Antonio for many helpful discussions. We are grateful to the members of the Aspen Center for Physics for their hospitality during our stay at Aspen where this work was begun.

\section{References}

1. Lehmann, H.: Nuovo Cimento 11, 342 (1954).

2. Wilson, K.: On products of quantum field operators at short distances. Cornell Report (1964).

3. - Phys. Rev. 179, 1499 (1969).

4. Valatin, J.: Proc. Roy. Soc. A 225, 535 and 226, 254 (1954).

5. Brandt, R.: Ann. Phys. 52, 122 (1969) and Fortschritte der Physik.

6. Zimmermann, W.: Commun. math. Phys. 6, 161 (1967), 10, 325 (1968).

7. Glimm, J., Jaffe, A.: Ann. Math. 91, 362 (1970).

8. Lowenstein, J.: Commun. math. Phys. 16, 265 (1970).

Wilson, K.: Phys. Rev. D, Oct. 15 (1970). 
9. Rosen, L.: The $\left(\varphi^{2 n}\right)_{2}$ quantum field theory, higher order estimates, NYU-preprint, to be published in Pure and Applied Mathematics.

Dimock, J.: Estimates, renormalized currents and field equations for the Yukawa field theory, Harvard Thesis, to be published.

10. Wilson, K.: Unpublished.

11. Zimmermann, W.: 1970 Brandeis lectures, Vol. I. Cambridge: MIT Press 1970.

12. Symanzik, K.: Cargese lecture notes and private communication.

Lowenstein, K.: Normal product quantization of currents in lagrangian field theory. Preprint University of Pittsburgh (1971).

Stora, R.: In preparation.

13. de Mottoni, R., Genz, H.: Nuovo Cimento 67 B, 1 (1970).

14. See, for instance, R. Streater and A. Wightman, PCT, Spin and Statistics. New York: Benjamin 1964.

W. Zimmermann

Department of Physics

New York University

251 Mercer Street

New York, N. Y. 10012, USA
Kenneth G. Wilson

Laboratory of Nuclear Studies

Cornell University

Ithaca, N.Y., USA 